\title{
Importância das condições de armazenamento de microgreens: Análise bibliométrica
}

\section{e revisão de literatura}

\author{
Importance of microgreen storage conditions: Bibliometric analysis and literature review \\ Importancia de las condiciones de almacenamiento de microverdes: análisis bibliométrico y \\ revisión de la literatura
}

Recebido: 05/02/2022 | Revisado: 10/02/2022 | Aceito: 13/02/2022 | Publicado: 20/02/2022

Tayná Josefa Bezerra

ORCID: https://orcid.org/0000-0001-9716-6450 Universidade do Estado de Mato Grosso, Brasil E-mail: tayna.bezerra@unemat.br

Thiaila Tamires da Silva

ORCID: https://orcid.org/0000-0002-0901-0710 Universidade do Estado de Mato Grosso, Brasil E-mail: thiaila.tamires@unemat.br

Raquel Aparecida Loss

ORCID: https://orcid.org/ 0000-0002-6022-7552 Universidade do Estado de Mato Grosso, Brasil E-mail: raquelloss@ unemat.br Claudineia Aparecida Queli Geraldi ORCID: https://orcid.org/0000-0001-5255-9752 Universidade do Estado de Mato Grosso, Brasil E-mail: claudineia.geraldi@unemat.br

Sumaya Ferreira Guedes

ORCID: https://orcid.org/0000-0002-1676-6030 Universidade do Estado de Mato Grosso, Brasil E-mail: sumaya.guedes@unemat.br

\begin{abstract}
Resumo
Microverdes são vegetais jovens que consistem basicamente de três partes: um caule central, folhas cotilédones e um par de folhas verdadeiras. Podem ser cultivados de modo orgânico e colhidos entre 1-2 semanas após a semeadura. Os microverdes concentram 40 vezes mais nutrientes que vegetais maduros, apresentando maior quantidade de nutrientes e micronutrientes. Porém, os microverdes enfrentam variados problemas de conservação e durabilidade devido ao seu difícil armazenamento, por possuir folhas frágeis, com facilidade de murcha e senescência precoce. Por isso, precisam de embalagens que facilitem sua respiração e mantenha sua qualidade visual, já que seu consumo muitas vezes é regido por sua aparência. Nesse contexto, o presente trabalho objetivou realizar uma análise bibliométrica e revisão de literatura acerca da importância do armazenamento de microgreens, trazendo estudos recentes com técnicas que tem sido estudadas e tem mostrado resultados promissores. Verificou-se que o uso de soluções cloradas e ácidos orgânicos tem grande potencial para aumentar a vida de prateleira dos microverdes, além disso, a maioria dos estudos utilizou embalagem de filmes OTR (taxa de transmissão de oxigênio), ou polietileno/embalagens a vácuo, com temperaturas de refrigeração variando de 1 a $10{ }^{\circ} \mathrm{C}$, onde a temperatura de $5{ }^{\circ} \mathrm{C}$ mostrou-se ser a mais ideal para aumentar a vida de prateleira da maioria dos microgreens.
\end{abstract}

Palavras-chave: Vegetais; Nutrientes; Vida de Prateleira.

\begin{abstract}
Microgreens are young plants that basically consist of three parts: a central stem, cotyledon leaves, and a pair of true leaves. They can be grown organically and harvested between 1-2 weeks after sowing. Microgreens concentrate 40 times more nutrients than mature vegetables, presenting a greater amount of nutrients and micronutrients. However, microgreens face various conservation and durability problems due to their difficult storage, as they have fragile leaves, with ease of wilting and early senescence. Therefore, they need packaging that facilitates their breathing and maintains their visual quality, since their consumption is often governed by their appearance. In this context, the present work aimed to carry out a bibliometric analysis and literature review about the importance of storing microgreens, bringing recent studies with techniques that have been studied and have shown promising results. It was found that the use of chlorinated solutions and organic acids has great potential to increase the shelf life of microgreens, in addition, most studies used OTR (oxygen transmission rate) film packaging, or polyethylene/vacuum packaging, with refrigeration
\end{abstract}


temperatures ranging from 1 to $10^{\circ} \mathrm{C}$, where the temperature of $5^{\circ} \mathrm{C}$ proved to be the most ideal to increase the shelf life of most microgreens.

Keywords: Vegetables; Nutrients; Shelf Life.

\section{Resumen}

Los microgreens son plantas jóvenes que básicamente constan de tres partes: un tallo central, hojas cotiledóneas y un par de hojas verdaderas. Se pueden cultivar orgánicamente y cosechar entre 1 y 2 semanas después de la siembra. Los microgreens concentran 40 veces más nutrientes que los vegetales maduros, presentando una mayor cantidad de nutrientes y micronutrientes. Sin embargo, los microvegetales enfrentan diversos problemas de conservación y durabilidad debido a su difícil almacenamiento, ya que tienen hojas frágiles, con facilidad de marchitamiento y senescencia temprana. Por ello, necesitan un envase que les facilite la respiración y mantenga su calidad visual, ya que su consumo muchas veces se rige por su apariencia. En este contexto, el presente trabajo tuvo como objetivo realizar un análisis bibliométrico y revisión bibliográfica acerca de la importancia del almacenamiento de microvegetales, trayendo estudios recientes con técnicas que han sido estudiadas y han mostrado resultados prometedores. Se encontró que el uso de soluciones cloradas y ácidos orgánicos tiene un gran potencial para aumentar la vida útil de los microvegetales, además, la mayoría de los estudios utilizaron empaques de película OTR (tasa de transmisión de oxígeno), o empaques de polietileno/vacío, con temperaturas de refrigeración que van desde 1 a $10{ }^{\circ} \mathrm{C}$, donde la temperatura de $5{ }^{\circ} \mathrm{C}$ resultó ser la más idónea para aumentar la vida útil de la mayoría de los microgreens.

Palabras clave: Hortalizas; Nutrientes; Duracion.

\section{Introdução}

Por serem ricos nutricionalmente e podendo ser cultivados de modo orgânico, os alimentos frescos tem ganhado espaço no mercado e no paladar do consumidor, como saladas e vegetais que podem ser consumidos in natura ou que podem ser facilmente cozidos (Choi et al., 2020). Cada vez mais a população está se conscientizando da necessidade do consumo de alimentos mais saudáveis, produtos como frutas e vegetais tem sido incluídos com maior frequência na alimentação da população (Freitas, 2020)

Microgreens são vegetais jovens colhidos entre 1-2 semanas após a semeadura. Eles consistem em três partes: um caule central, folha ou folhas cotilédones e um par de folhas verdadeiras. São diferentes de brotos ou verduras porque são colhidos sem as raízes. O interesse em microgreens aumentou por conter maiores quantidades de nutrientes e micronutrientes que promovem a saúde em comparação com suas contrapartes maduras. Por serem tão ricos em nutrientes, quantidades menores podem fornecer benefícios nutricionais semelhantes a quantidades maiores de vegetais maduros. Além disso, a composição química dos microgreens difere marcadamente de sua forma madura. (Lopez-Cervantes et al., 2013)

Os microverdes concentram 40 vezes mais nutrientes que vegetais maduros, por isso são considerados superalimentos, e também são ótimas fontes de vitamina C. Por exemplo, o microverde de repolho roxo que possui alto teor de minerais, como fósforo, potássio, magnésio, zinco, ferro, cálcio, sódio e cobre, e os microverdes de brócolis que tem entre 1,15 a 2,32 vezes mais minerais do que o próprio brócolis maduros (Laurindo, 2020).

Apesar de seus diversos benefícios, os microverdes enfrentam variados problemas de conservação e durabilidade devido ao seu difícil armazenamento, por possuir folhas frágeis, com facilidade de murcha e senescência precoce. Por isso, precisam de embalagens que facilitem sua respiração e mantenha sua qualidade visual, já que seu consumo muitas vezes é regido por sua aparência (Turner et al., 2020).

Os microverdes ainda são novidades no Brasil, seja para os consumidores quanto para os pequenos produtores rurais. Além disso, os parâmetros de umidade e temperatura variam de acordo com cada estado. Assim, torna-se necessário a realização de pesquisas que avaliem as melhores condições de conservação adaptadas para essa região, agregando também estratégias adequadas de pré e pós-colheita, de modo a permitir que os microverdes mantenham o maior nível possível de seu valor nutricional (Castellino et al., 2018). 
Nesse contexto, o presente trabalho objetivou realizar uma análise bibliométrica e revisão de literatura acerca da importância do armazenamento de microgreens, trazendo estudos recentes com técnicas que tem sido estudadas e tem mostrado resultados promissores.

\section{Metodologia}

De acordo com Prodanov e Freitas (2013), esta pesquisa enquadra-se como qualitativa, quanto à abordagem, e bibliográfica e documental, conforme os procedimentos. O levantamento dos artigos baseou-se na busca de documentos a partir da inserção de determinados termos na base de dados Scopus. Inicialmente, foram pesquisados artigos que abordassem no título, resumo e palavras-chave os termos "MICROGREENS" AND "STORAGE”, totalizando 26 artigos.

Os resultados das buscas preliminares no Scopus foram analisados para eliminar artigos com conteúdo sobreposto, para recategorizar determinados artigos ao método considerado mais adequado com base em seu conteúdo, além disso, para excluir artigos que não se enquadrassem no escopo deste artigo. Após leitura preliminar dos abstracts dos artigos, foram selecionados 20 artigos para o desenvolvimento do estado da arte desta revisão.

\section{Análise Bibliométrica}

De acordo com a análise bibliométrica realizada, na Figura 1, nota-se que tema sobre armazenamento de microgreens é pouco estudado, com publicações iniciadas em 2012, e grande instabilidade de publicação de artigos até o presente momento. Tal fato fica mais evidente ao observamos o maior pico de publicação, registrado no ano de 2020, com apenas 6 artigos.

Figura 1: Produção científica anual de artigos sobre armanenamento de microgreens.

\section{Annual Scientific Production}

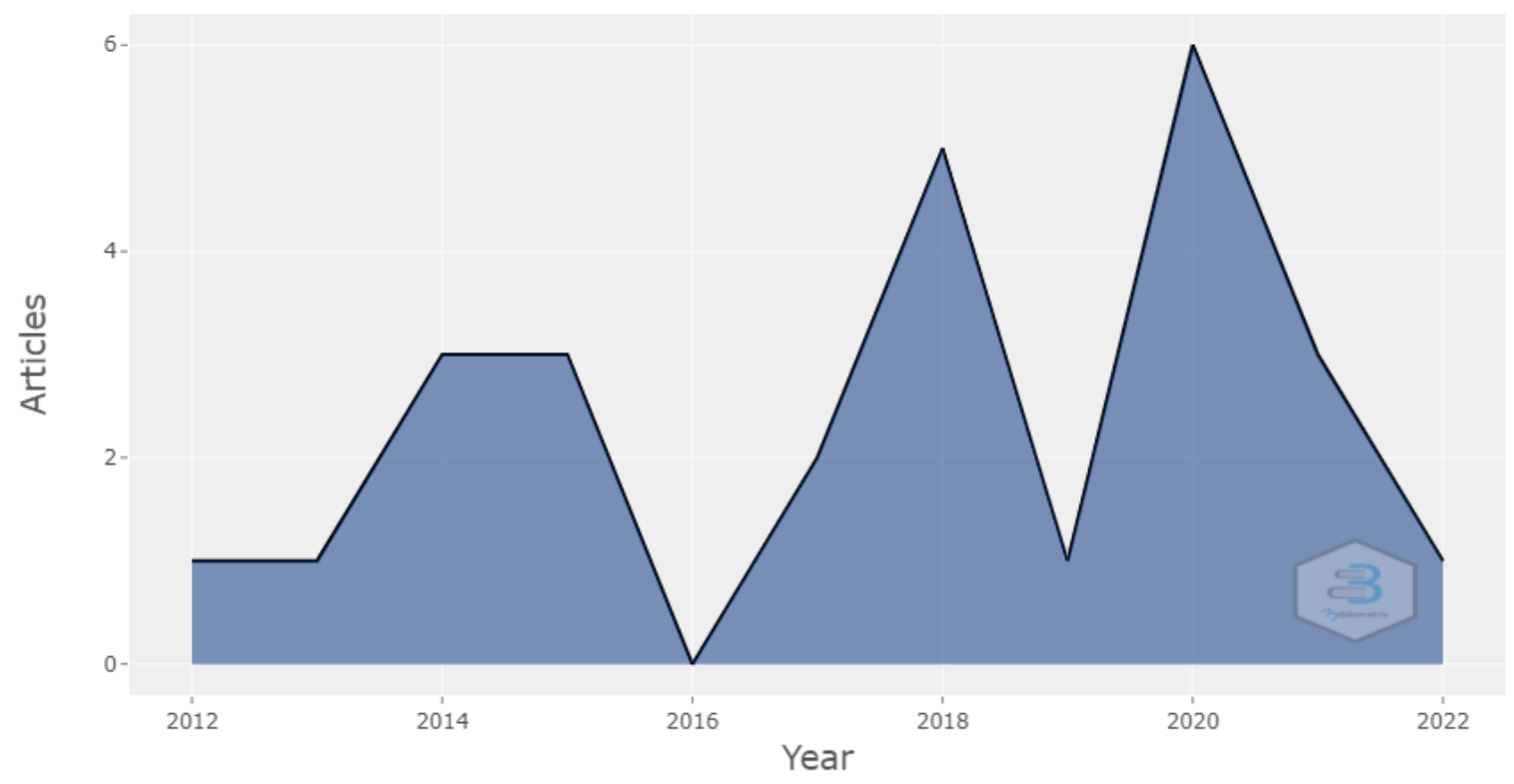

Fonte: Bibliometrix - RStudio (2021).

Com relação à produção científica anual por país, nota-se que os Estados Unidos é o país que mais publica artigos sobre armazenamento de microgreens, seguido da China, Turquia, Índia e Tailândia (Figura 2). 
Figura 2: Produção científica anual de artigos sobre armanenamento de microgreens por país.

\section{Country Scientific Production}

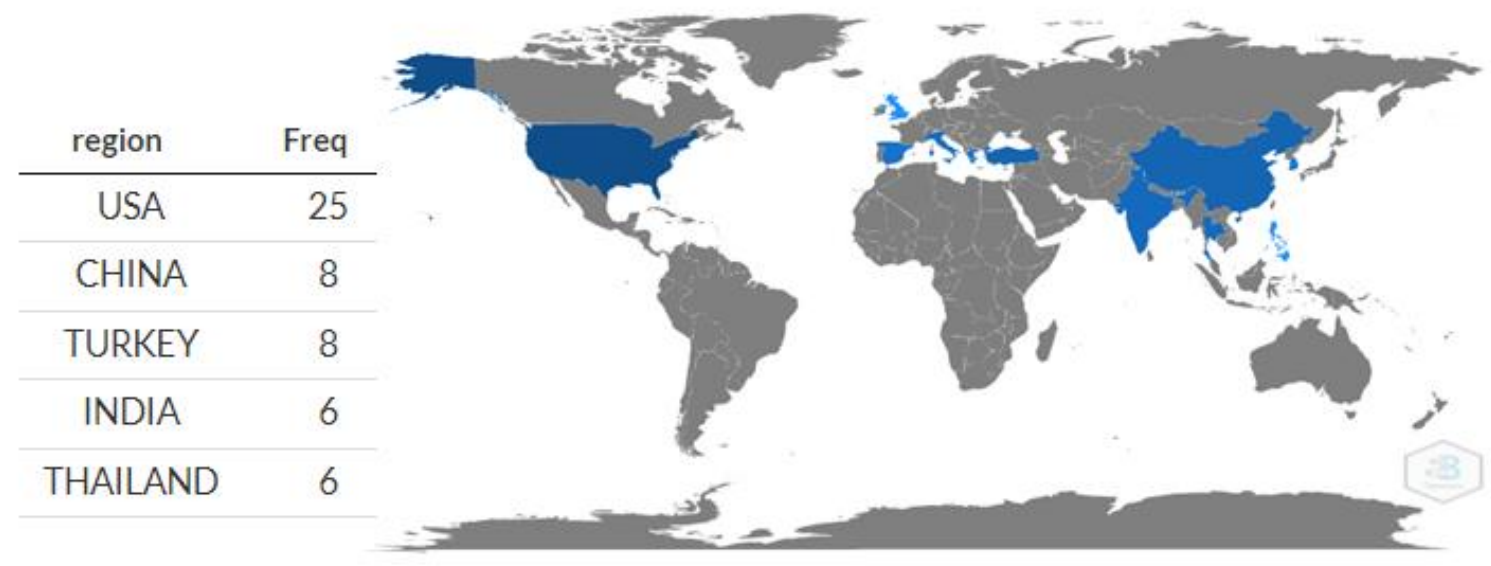

Fonte: Bibliometrix - RStudio (2021)

A Figura 3, apresenta a nuvem de palavras gerada com as 50 palavras mais citadas nos abstract dos artigos analisados neste review, em que as palavras postharvest, study, control e results sugerem os estudos analisados e seus resultados a respeito da pós-colheita e métodos de controle de microrganismos indesejáveis, enquanto que as palavras microgreen, fresh, storage, quality e shelf indicam os principais tópicos correlacionados com o tema.

Figura 3: Nuvem de palavras com as 50 palavras mais citadas nos abstract dos artigos.

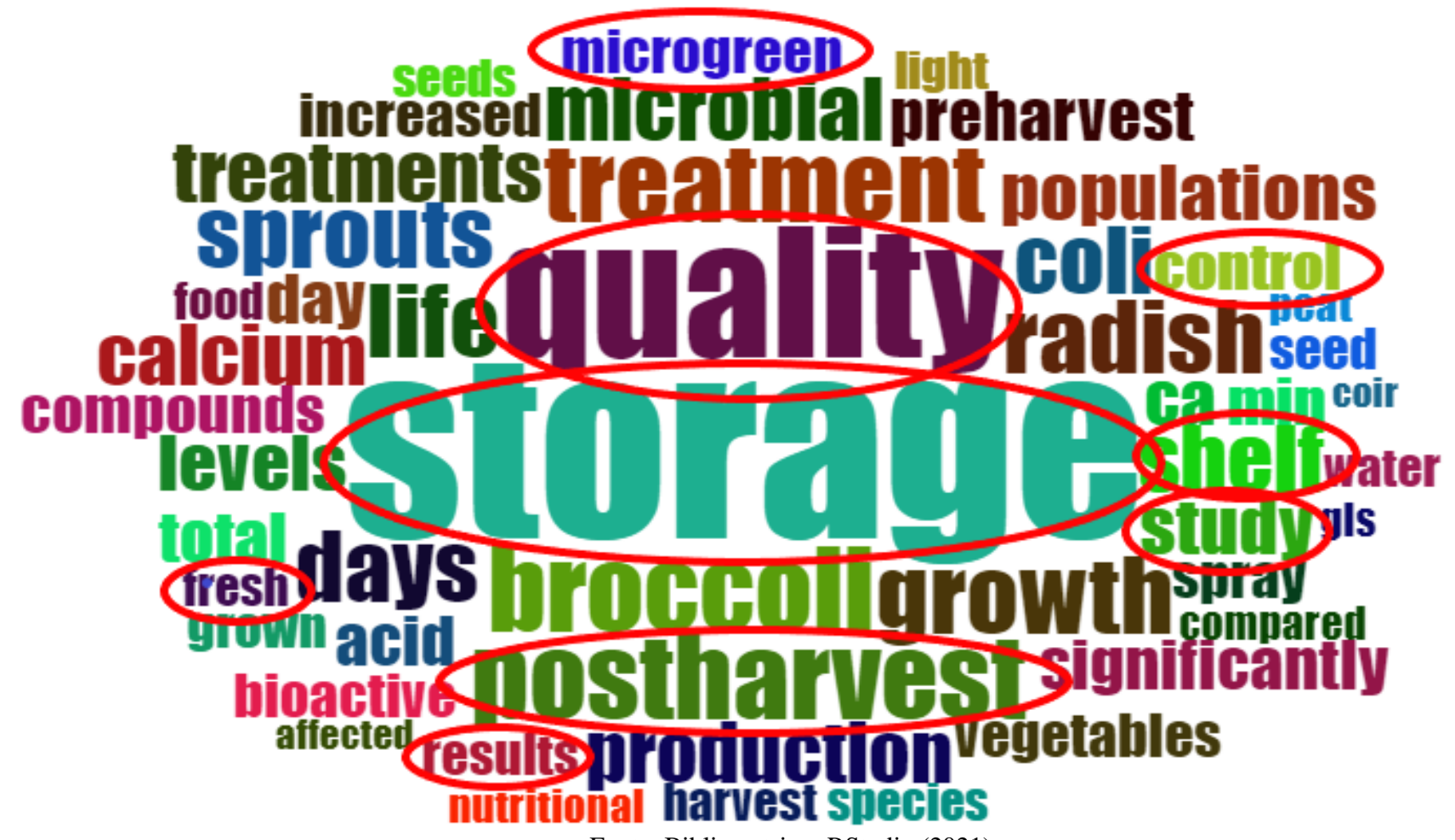

Fonte: Bibliometrix - RStudio (2021). 
Por fim, na Figura 4 é apresentado as 10 palavras com maior crescimento ao longo do tempo citadas nos títulos dos artigos, assim, as palavras "SHELF" "LIFE" (Vida de prateleira) "POSTHAVERST" (Pós-colheita) e "STORAGE" (Armazenamento), reforçam a importância de pesquisas para controle da vida pós-colheita dos microgreens, favorecendo o aumento da vida de prateleira.

Figura 4: Crescimento das palavras citadas nos titulos dos artigos (2012-2021).

\section{Word Growth}

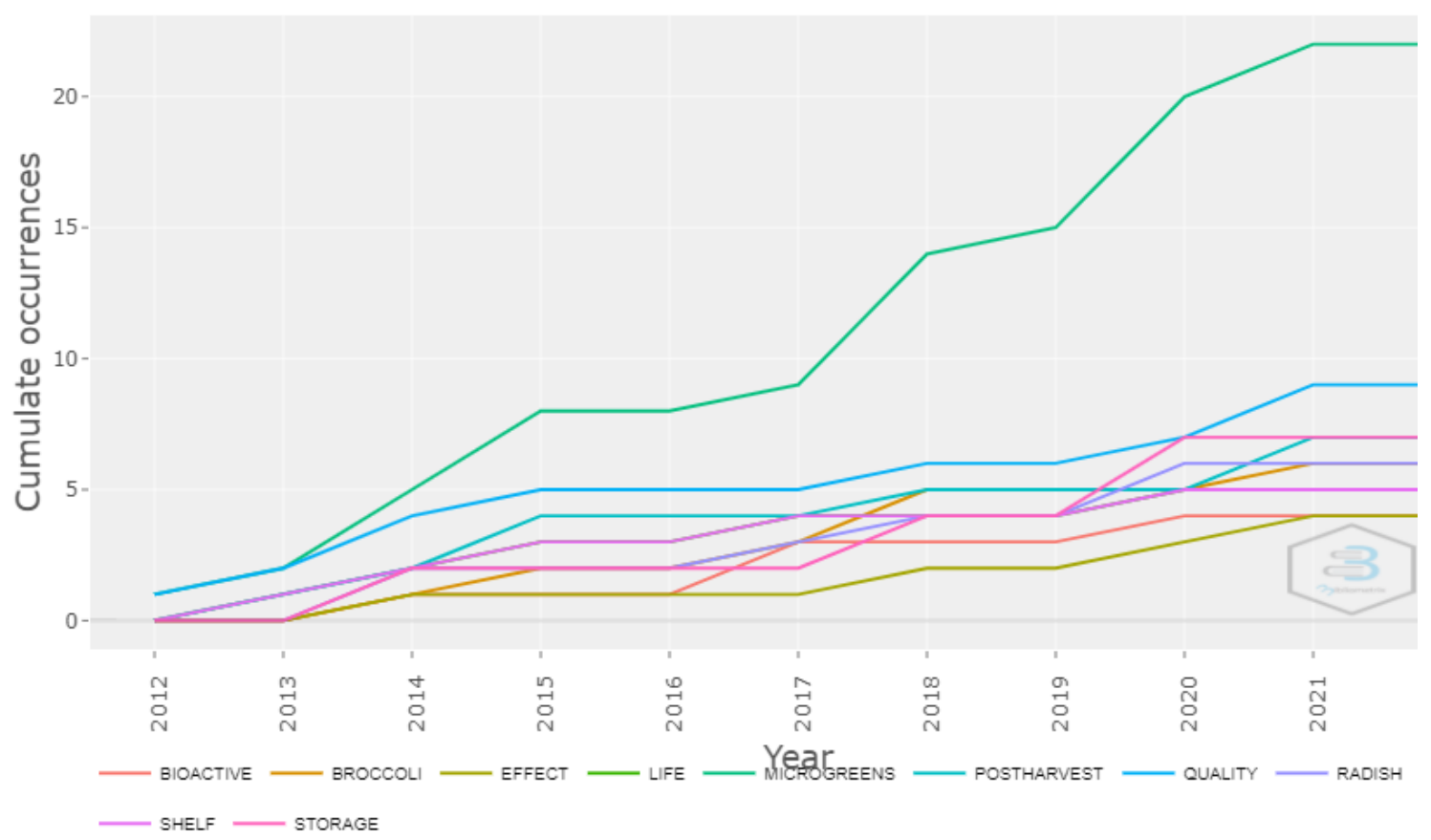

Fonte: Bibliometrix - RStudio (2021).

\section{Revisão de Literatura}

\subsection{Microverdes / Microgreens}

Os microgreens ou microverdes são plantas jovens comestíveis, tida como uma opção menor de plantas já conhecidas, são oriundas comumente de sementes e os mais consumidos são os vegetais, cereais e ervas (Katsenios et al., 2021). Conhecido como uma planta pequena, com comprimento variando de 5 a $10 \mathrm{~cm}$ e de vida útil curta, entre 10 a 14 dias (Rocchetti et al., 2020).

Estima-se que por volta da década de 1980 surgiu as primeiras iguarias utilizando microverdes nos cardápios dos restaurantes sofisticados californianos (Verlinden, 2020). Um modo de diferenciar microverdes de brotos e baby leaf é através do seu período de desenvolvimento. Brotos possui um crescimento breve, podendo variar de 4 a 10 dias e as folhas bebês um período de desenvolvimento mais longo, variando entre 20 e 40 dias (Katsenios et al., 2021).

Além disso, os microverdes são uma fonte de vários nutrientes importantes para o ser humano, como carotenoides, fenólicos, vitaminas, minerais, tocoferóis, ácido ascórbico, etc (Katsenios et al., 2021; Paradiso et al., 2018) e ficaram mundialmente conhecidos devido a sua textura crocante, cores atrativas, aromas e sabores diversos (Katsenios et al., 2021; Verlinden, 2020).

Um fitonutriente muito importante presente nos microgreens é o gluco-silonato (GLS), que é amplamente associado a prevenção do câncer, diminuição dos níveis de colesterol e desintoxicação dos alérgenos (Lu et al., 2018). 
A produção dos microverdes é comumente realizada em solo ou algum substituto colocado bandejas em estufas, sendo alguns dos substratos conhecidos são: turfa, fibra de coco, lã mineral, casca de coco em pó, vermiculita, perlita, etc. A turfa é a mais utilizada em alguns países, mas por possuir um valor econômico alto, foi sendo substituída em outros lugares por fibra de coco, de modo isolado ou combinado com outros meios de plantio. Esses meios de cultivo devem ser esterilizados de modo adequado antes do plantio para evitar a ação microbiana e melhorar o desenvolvimento dos microgreens (Photchanachai; et al., 2018).

Figura 5: Modelo de produção de microverdes em sistema caseiro.
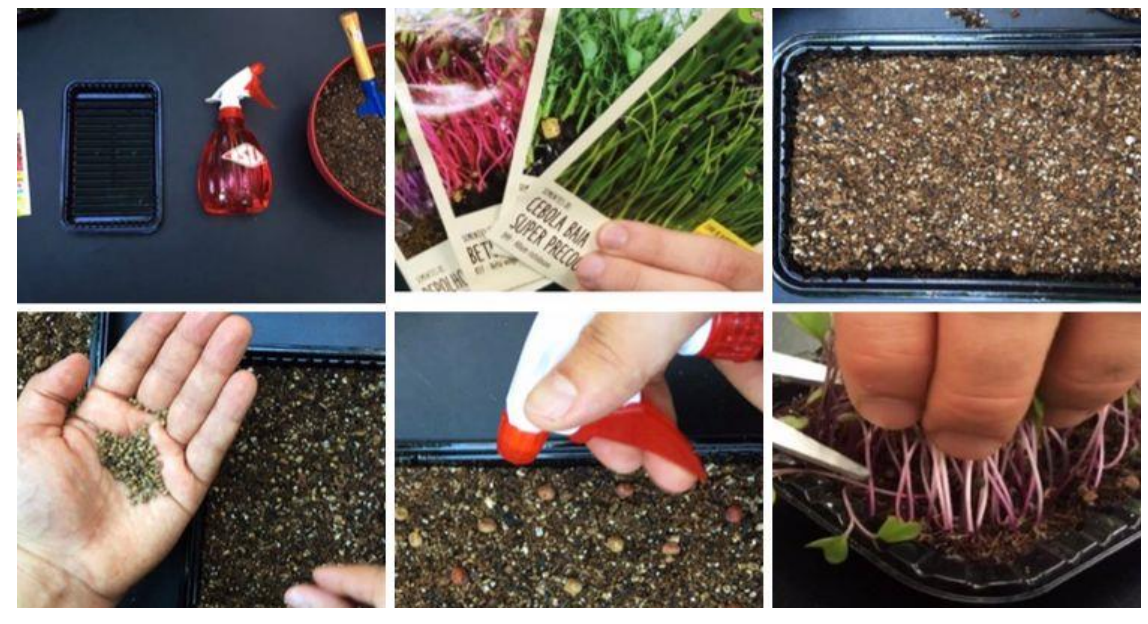

Fonte: Site Vamos Comer Melhor (2019).

Conforme mostrado na Figura 5, os microgreens podem ser cultivados em locais de pouco espaço, como meios urbanos, por ser um cultivo que não exige um sistema complexo para produzir (Renna \& Paradiso, 2020). Assim como os brotos, antes de serem postas em solo, as sementes de microverdes podem ser embebidas em água no período noturno para aumentar sua taxa de germinação. Passados 3 dias são postas em exposição a luz sendo regadas diariamente até a emissão das primeiras folhas. Deve-se tomar cuidado quanto a qualidade da semente utilizada e realizar trocas da água constantemente para não favorecer a propagação de agentes microbiológicos (Turner et al. 2020).

Além disso, o modo de cultivo pode ser feito em câmaras sob luz artificial ou cultivados em estufas, sendo esse último geralmente mais aderido para escalas de produção, onde chegam a apresentar um índice de rendimento de $90 \mathrm{~kg}$ de microverdes por semana em uma área de $400 \mathrm{~m}^{2}$, podendo empregar de 3 a 4 pessoas. Lembrando que, essa proporção de rendimento é variável e irá depender de fatores como temperatura, época de plantio, qualidade das sementes e espécie plantada, umidade, irrigação, fotoperíodo, manejo, entre outros (Verlinden, 2020).

Em câmaras de luz artificial, as luzes de LED apresentam maiores vantagens em comparação a outros tipos de iluminação, fótons entre 150 e $440 \mu \mathrm{mol} \mathrm{m} / \mathrm{s}^{-2}$ mostraram ser ideais para grande parte dos microverdes (Verlinden, 2020). Isso se deve ao fato das luzes de LED permitir maior uniformidade na distribuição de luminosidade comparada a outras lâmpadas mais convencionais (Turner; Luo; Buchanan, 2020). Uma outra observação é a luz azul favorece o crescimento e concentrações ótimas de antioxidantes, já em alta luz e UVA parece acumular os antioxidantes (Verlinden, 2020).

O processo de germinação dos microverdes duram em torno de 08 a 21 dias, com variação dependente da espécie plantada, conforme a Tabela 1 . 
Tabela 1: Tempo de crescimento de diferentes microverdes.

\begin{tabular}{ccc}
\hline Microverde & Tempo médio de crescimento & Referência \\
\hline Alface & 21 dias & Byrdwell, 2021 \\
Brócolis & 14 dias & Lu et al. 2021 \\
Beterraba & 10 dias & Rocchetti et al. 2021 \\
Rabanete & 14 dias & Baenas et al. 2017 \\
Coentro & 10 dias & Binder, 2012 \\
\hline
\end{tabular}

Fonte: Autores.

Durante a comercialização é indicada que os produtos sejam mantidos em sistema de refrigeração com temperaturas de $5^{\circ} \mathrm{C}$ e $10^{\circ} \mathrm{C}$. (Scott \& Mayer, 2017).

Em geral, as famílias de Brassicaceae, Asteraceae, Chenopodiaceae, Lamiaceae, Apiaceae, Amarillydaceae, Amaranthceae e Cucurbitaceae possuem as espécies mais exploradas (Kyriacou et al., 2016). As cultivares da família Brassicaceae são tidas como as mais produzidas mundialmente, dando destaque para hortaliças como brócolis, couve, rabanete, couve-flor, repolho, rúcula, entre outras (Di Bella et al., 2021).

A maioria dos estudos a respeito dos microgreens são datadas dos últimos 7 anos, mas demonstra um grande avanço em pesquisa (Turner; Luo; Buchanan, 2020). No Brasil, apesar da facilidade de cultivo, os microverdes ainda são uma novidade, seja para o agricultor ou consumidores.

\subsection{Problemas fitossanitários}

Os microverdes enfrentam muitos problemas, principalmente relacionado a seu armazenamento, podendo se decompor rapidamente, gerando odor e sabor desagradável, descoloração ou até amolecimento/apodrecimento dos tecidos. Esses efeitos indesejáveis são causados, em muitas vezes, por microrganismos que se multiplicaram facilmente devido ao ambiente que se tornou favorável. Para evitar a propagação desses microrganismos e manter as qualidades dos microgreens, faz-se uso de sanitizantes, individuais ou combinados, como os ácidos orgânicos (cítrico e ascórbico), etanol em spray e cloro, sendo esse último o mais comum (Mir et al., 2016).

Diferente dos brotos, os microverdes, até agora, não apresentaram nenhum surto de origem alimentar, mas possui riscos relacionados a contaminação de sementes. Por isso, é recomendado ter precauções sanitárias visando a eliminação de patógenos em sementes, como uso de tratamentos eficazes (químicos ou orgânicos), podendo ser utilizados os mesmos indicados para brotos, e o teste de germinação preliminar (Kyriacou et al., 2016).

Os surtos alimentares relacionados aos brotos foram, em sua maioria, causados por Salmonella spp., Escherichia coli O157: H7 e Listeria monocytogenes, entre outros (Baenas et al., 2017). Esses mesmos patógenos podem se apresentar em microverdes desde seu cultivo até comercialização, por ser consumido da mesma forma que os brotos, geralmente cru, e possuir o mesmo crescimento rápido (Işs1k et al., 2020; Reed et al., 2018). A Salmonella spp. pode ser mais problemática em cultivos hidropônicos, mas para chegar em uma conclusão mais exata muitos estudos ainda devem ser realizados (Reed et al., 2018).

Dessa forma, os microverdes são tidos como mais seguros que os brotos, já que, por exemplo, a Escherichia coli 0157:H7 se manifesta em maior quantidade nos cotilédones das plantas e vai diminuindo gradativamente ao longo do seu crescimento, sendo assim, é menor em plantas que já apresentam folhas verdadeiras (Verlinden, 2020).

Estudos recentes demonstraram que plantas oriundas de sementes inoculadas com Escherichia coli quando expostas em luzes fluorescentes podem acentuar a eliminação da bactéria (Turner et al., 2020). Uma vez contaminado, é quase impossível a remoção do patógeno na planta, pois coloniza-se rapidamente sua parte aérea, principalmente se houver algum ferimento presente. Outro fator é o uso limitado de métodos de tratamento, devido a produção que por muitas vezes é feita de modo orgânico (Işık et al., 2020). 
O saneamento é o fator principal para diminuir as condições favoráveis de desenvolvimento microbiológico, além disso resulta na exclusão da maioria dos pesticidas no plantio de Microgreens, em caso de cultivo inorgânico (Verlinden, 2020).

Os substitutos do solo, como a fibra de coco, se fervida como método de esterilização pode diminuir a propagação microbiana por até 3 dias, se estendido o tempo de fervura por mais 30 minutos reduz ainda mais no substituto do solo, mas não nos microgreens (Photchanachai et al., 2018).

Por tratar-se de plantas jovens e comestíveis, os microverdes limita o uso de fungicidas como método de controle, por isso muitos estudos tem sido desenvolvidos testando métodos de controle biológico, como o uso do fungo Trichoderma e bactérias endofíticas do gênero Herbaspirillum (Verlinden, 2020).

\subsection{Cuidados durante a colheita e condições de armazenamento}

Muitos estudos estão sendo desenvolvidos a respeito da colheita de microverdes de modo mecanizado, até então o cultivo vem sendo feito de modo manual e seu armazenamento é feito em potes ou sacos plásticos. Sobre as condições de preservação do alimento, deve-se considerar a fragilidade dos microverdes e sua fácil deterioração, respeitando suas exigências para manter-se fresca, relacionadas a temperatura, luz e taxas de oxigênio. Estudos demonstraram que o uso de baixas temperaturas, luz e atmosfera modificada são fatores contribuintes para a boa qualidade de alguns microgreens em embalagens (Verlinden, 2020).

A colheita é feita através do corte dos caules, pois suas raízes não são consideradas comestíveis. O cuidado em relação ao corte deve ser destacado, por acelerar a deterioração e desidratação, fazendo com que o produto perca qualidade em comercialização (Turner et al., 2020). Pois, mesmo após o corte os microverdes continuam seus processos biológicos, como respiração e transpiração (Rocchetti et al., 2020).

No caso da colheita, muitos fatores devem ser respeitados, como o tempo ideal de maturação de cada espécie, evitar lesões ao manuseio, o responsável por fazer a coleta deve estar habilitado, recipientes e instrumentos reutilizáveis devem ser limpos e higienizados, como os utensílios de corte, e devem ser mantidos armazenados longe do local de cultivo, evitando a contaminação cruzada. Uma boa opção para evitar transtornos relacionados a colheita, seria comercializar os microverdes enraizados em seu local de crescimento (Turner et al., 2020).

A refrigeração e escolha da embalagem interferem no produto e são essenciais para sua conservação. É sugerido temperatura de $5{ }^{\circ} \mathrm{C}$, alta umidade e o uso de embalagem com atmosfera modificada podendo equilibrar vida útil do produto e sua qualidade. A atmosfera modificada mantém a taxa de umidade e respiração ideal, evitando deterioração e propagação de patógenos (Turner et al., 2020).

Dalal et al. (2020) verificaram que os microgreens de girassol submetidos a pesquisa de qualidade pós-colheita utilizando soluções de ácidos orgânicos prolongar sua vida útil de prateleira. Já as soluções com etanol inicialmente tiveram bons resultados e posteriormente a alta concentração favoreceu o crescimento microbiológico, devido a danificação dos tecidos superficiais da planta.

Valores fenólicos podem variar no período de armazenamento, seus valores melhores alcançados são após 10 dias. Além disso, efeitos como tempo, condições de armazenamento e mudanças que ocorrem durante a digestão podem medir o valor funcional dos microverdes (Rocchetti et al., 2020). Assim, pesquisas são necessárias a fim de promover a alta preservação desses e outros nutrientes dos microverdes pós-colheita (Paradiso et al., 2018). 


\subsection{Avanços nos estudos sobre armazenamento de microgreens}

Na Tabela 2, são apresentados estudos recentes que objetivaram avaliar condições e técnicas para armazenamento de microgreens, bem como os principais resultados obtidos em cada estudo específico.

Tabela 2: Condições e técnicas avaliadas em estudos recentes para armazenamento de microgreens.

\begin{tabular}{|c|}
\hline Título do estudo \\
\hline $\begin{array}{l}\text { Microverdes de Beterraba vermelha (Beta } \\
\text { vulgaris) e amaranto (Amaranthus sp.): } \\
\text { Efeito do armazenamento e digestão } \\
\text { gastrointestinal in vitro no perfil } \\
\text { metabolômico não-acompanhado. }\end{array}$ \\
\hline \begin{tabular}{lccc} 
Crescimento & de & \multicolumn{2}{c}{ Escherichia } \\
coli e Listeria & \multicolumn{2}{c}{ monocytogenes em } \\
microverdes de rabanete lavados com \\
hipoclorito de sódio durante o \\
armazenamento.
\end{tabular} \\
\hline $\begin{array}{l}\text { Efeito do spray de } \mathrm{CaCl}_{2} \text { pré-colheita e } \\
\text { radiação UV-B pós-colheita na qualidade } \\
\text { de armazenamento de microverdes de } \\
\text { brócolis, uma fonte mais rica de } \\
\text { glicosinolatos (GLs) }\end{array}$ \\
\hline $\begin{array}{l}\text { Efeito da exposição à luz na qualidade } \\
\text { sensorial, concentrações de bioativos } \\
\text { compostos e capacidade antioxidante de } \\
\text { microverdes de rabanete durante } \\
\text { armazenamento de temperatura }\end{array}$ \\
\hline
\end{tabular}

Qualidade pós-colheita e vida útil de microverdes de rabanete impactadas por temperatura de armazenamento, filme de embalagem e tratamento de lavagem com cloro.

Produção de microverdes de espécies de plantas locais na Tailândia

Mudanças na população microbiana e qualidade dos microverdes tratados com diferentes desinfetantes e filmes de embalagem

Biologia pós-colheita, qualidade e vida útil de microverdes de trigo sarraceno

Qualidade pós-colheita de microverdes de girassol influenciada por ácidos orgânicos e tratamento com etanol

\footnotetext{
A aplicação de UVB pré-colheita aumenta o conteúdo de glicosinolato e melhora a qualidade pós-colheita de microverdes de brócolis
}

\section{Principais resultados}

Os perfis de polifenóis e betalaínas variaram significativamente entre as espécies de microgreens.

Ambos os microgreens forneceram atividades antioxidantes que aumentaram durante o armazenamento.

A digestão in vitro afetou fortemente a composição fitoquímica dos microgreens.

$\mathrm{O}$ tratamento com $\mathrm{NaOCl}(150 \mathrm{mg} / \mathrm{L}, 5 \mathrm{~min})$ reduziu significativamente $(2,3$ $\log \mathrm{UFC} / \mathrm{g}$ ) as populações de ambas as bactérias $(\mathrm{p}<0,05)$. Após a lavagem, os níveis de bactérias em microgreens de rabanete tratados com $\mathrm{NaOCl}$ foi avaliado durante o armazenamento a $4{ }^{\circ} \mathrm{C}, 10{ }^{\circ} \mathrm{C}, 15^{\circ} \mathrm{C}$ e $20^{\circ} \mathrm{C}$ por 3 dias. Os crescimentos de E. coli e L. Monocytogenes aumentou rapidamente em temperaturas acima de $15^{\circ} \mathrm{C}$ e $10^{\circ} \mathrm{C}$, respectivamente, a partir do dia 1.

O GLs alifático total em microgreens de brócolis foi muito maior do que em brócolis maduros. Os níveis de GLs aumentaram significativamente após a pulverização pré-colheita com $10 \mathrm{mM}$ de $\mathrm{CaCl} 2$.

A radiação UV-B pós- colheita não alterou os níveis de GLs alifático total em microgreens.A pulverização de cálcio pré-colheita resultou em maior qualidade visual geral e maior vida útil de armazenamento.

Os microverdes de rabanete armazenados em local escuro mantiveram sua qualidade, enquanto aqueles expostos a luz deterioraram rapidamente ambos expostos por 16 dias a uma temperatura de $5{ }^{\circ} \mathrm{C}$. Sacos OTR (taxa de transmissão de oxigênio) contribuíram para manter a qualidade do produto ao contrário dos LMP (microperfurados a laser)

A temperatura de $1{ }^{\circ} \mathrm{C}$ mostrou ser ideal para o armazenamento de microverdes de rabanete, já que em conjunto com as embalagens de sacos de filme OTR (taxa de transmissão de oxigênio) mantiveram a vida útil dos microgreens por até 28 dias. A temperatura de $5{ }^{\circ} \mathrm{C}$ mostrou médio desempenho e a temperatura de $10^{\circ} \mathrm{C}$ prejudicou a qualidade do produto. $\mathrm{O}$ tratamento com cloro teve eficiência inicialmente no combate a atividade microbiana, mas passados 7 dias houve proliferação microbiana.

$\mathrm{O}$ armazenamento dos microverdes, após lavagem com solução de cloro e mantido em refrigeração por 7 dias a $5^{\circ} \mathrm{C}$, foi capaz manter um nível seguro das populações de leveduras, mofos, Salmonella e E. coli.

O tratamento com ácido cítrico + etanol 50\% mostrou ser uma alternativa de substituição do uso do cloro como sanitizante e as embalagens de polietileno foram mais eficientes que os sacos de polipropileno.

Temperatura de $5{ }^{\circ} \mathrm{C}$ em sacos de filme OTR (taxa de transmissão de oxigênio) obtiveram um desempenho ideal durante os 21 dias em que os microgreens de trigo sarraceno estiveram armazenados em refrigeração. Porém, a temperatura de $1{ }^{\circ} \mathrm{C}$ causou lesão por frio. No dia 0 as soluções cloradas reduziram a população microbiana, mas após 7 dias foi recuperada, devido à alta umidade após a lavagem. Mesmo com esses resultados, o uso da solução clorada $(50 \mathrm{mg} / \mathrm{L}$ e $100 \mathrm{mg} / \mathrm{L})$ se destacou comparado a lavagem feita com água.

Os microgreens de girassol foram armazenados em bandejas de poliestireno embrulhadas com plástico filme com 6 perfurações, papel filtro encharcado com água destilada foi usado para manter umidade e a temperatura da incubadora, variando de $10^{\circ} \mathrm{C} \pm 1{ }^{\circ} \mathrm{C}$. As soluções AA e CA + AA (ácido ascórbico e ácido cítrico + ácido ascórbico) obtiveram maior vida útil até o $12^{\circ}$ dia do experimento. No $16^{\circ}$ dia essas mesmas soluções apresentaram melhor preservação comparada as outras (vapor de etanol [EV], ácido cítrico $[\mathrm{CA}]$ e CA + etanol [E]).

Os microverdes de brócolis passaram pelos tratamentos pré-colheita de irradiação $0,27 \mathrm{Wh} / \mathrm{m}^{2} \mathrm{UVB}$ (raios ultravioleta $\mathrm{B}$ ) em conjunto com $10 \mathrm{mM}$ $\mathrm{CaCl}_{2}$ (cloreto de cálcio) em spray. Em resultado, houve o aumento das concentrações dos antioxidantes GLs (glicosinolatos) em cerca de $70 \%$

\section{Referência}

Rocchetti et

al.

(2020)

Choi et al.

Lu et al.

(2018)

Xiao et al

(2014b)

Xiao et al.

(2014a)

Muchjajib et

al. (2015)

Chandra, Kim

e Kim (2012)

Kou et al.

(2013)

Dalal,

Siddiqui e

Phogat

(2020)

Lu et al.

(2021) 
Caracterização nutricional e vida útil dos microverdes embalados comparado a microverdes sem tratamento. O armazenamento durou 21 dias, ocorreu menores valores de vazamento de eletrólitos nos tecidos e odores desagradáveis, mantendo assim de modo geral a qualidade do produto. Nos resultados pós-colheita houve a redução dos níveis de MY (mirosinase), gene responsável pela quebra do GL durante o armazenamento.

O estudo conduziu os microgreens das espécies de chicória, alface e brócolis para uma análise nutricional. Foram separados dois genótipos de cada espécie. Chicória: Molfetta (CM) e Italico a costa rossa (CR). Alface: Bionda da taglio (LB) e Trocadero (LT). Brócolis: Mugnuli (BM) e Natalino (BN). $\mathrm{CM}$ e BM são variedades locais de Puglia - Itália. Todos os microverdes, exceto LB, demonstraram ser boas fontes de Ca (cálcio). LT e CM mostraram ser boas fontes de $\mathrm{K}$ (potássio, LB e os microgreens de brócolis de vitamina $\mathrm{E}$ e as alfaces de carotenoides. Ambos os microverdes que foram cortados e armazenados em sacos de polipropileno a $5{ }^{\circ} \mathrm{C}$, e os autores verificaram que a vida útil dos microgreens avaliados não foi superior a 10 dias.

Foi utilizado a fibra de coco como um meio de cultivo para os microverdes de couve chinesa. Antes do plantio as fibras passaram por fervura de $30 \mathrm{~min}$ ou foram cozidas em vapor por 15 e 30 minutos. Os microverdes plantados na fibra cozida em vapor por 15 min mostraram tamanhos mais uniformes, assim como os microverdes sem tratamento, e houve redução de $6 \%$ de incidência de doenças, também teve menor população microbiana nos três primeiros dias. Embalagens clamshell fechadas e perfuradas diminuíram a perda de peso dos microverdes por até 9 dias em temperatura de $4{ }^{\circ} \mathrm{C}$ em todos os tratamentos. $\mathrm{O}$ estudo mostrou que a atividade antioxidante (DPPH) variou apenas no $14^{\circ}$ dia de armazenamento nas fibras esterilizadas por 30 $\min$.

As hortaliças foram mergulhadas em água e 0,05 ou $0,01 \mu \mathrm{M}$ de cianocobalamina (Vitamina $\mathrm{B}_{12}$ ) durante cinco minutos e posteriormente armazenadas durante 9 dias em temperatura de $4^{\circ} \mathrm{C} \pm 1^{\circ} \mathrm{C}$. O estudo mostrou que durante o armazenamento todas as hortaliças mergulhadas nas baixas concentrações de cianocobalamina apresentaram melhora na capacidade antioxidante e alguns compostos bioativos, como flavonoides e fenóis totais. Os microverdes mergulhados a $0,01 \mu \mathrm{M}$ tiveram aumento de betacianina, betaxantina e betalaínas totais.
Paradiso et al.

Photchanacha

i et al. (2018)

Supapvanich et al. (2020) para consumo (girassol e daikon) e micorverdes (amaranto vermelho) durante o armazenamento

Fonte: Autores.

Na Tabela 2, observa-se que as soluções cloradas, assim como os ácidos orgânicos, mostraram ter grande potencial para aumentar a vida de prateleira dos microverdes, mas apenas no período inicial do armazenamento, pois no decorrer dos dias a alta umidade favoreceu o crescimento da população microbiana, podendo danificar os microgreens e trazer riscos à saúde.

Verificou-se também que a maioria dos estudos utilizou embalagem de filmes OTR (taxa de transmissão de oxigênio), ou polietileno/embalagens a vácuo, com temperaturas de refrigeração variando de $10^{\circ} \mathrm{C}$ a $1^{\circ} \mathrm{C}$, onde a temperatura de $5{ }^{\circ} \mathrm{C}$ mostrou-se ser a mais ideal para aumentar a vida de prateleira da maioria dos microgreens.

\section{Considerações Finais}

Por possuir rápida deterioração, os microgreens necessitam de auxílio para manter um período mais prolongado de vida útil de prateleira, portanto, deve-se evitar que os microverdes colhidos não sofram transtornos relacionados a colheita, e que seja comercializado enraizados em seu local de crescimento, com tudo, a providência de um método que o cliente mantenha o produto com maior período em sua casa até o real consumo. $\mathrm{O}$ armazenamento de microverdes em ambiente de baixas temperaturas mostrou ser de grande vantagem, já que muitos estudos demonstraram sua eficiência e desempenho de qualidade ao uso de baixas temperaturas, juntamente com luz e atmosfera modificada são fatores contribuintes para a boa qualidade de alguns microgreens em embalagens. 
Visando a qualidade final do produto é essencial um local com baixa umidade, uma temperatura de em torno de $5^{\circ} \mathrm{C}$ e o uso de embalagem com taxa de respiração ideal para que não tenha interferência na qualidade do produto e atividade de população microbiana.

No geral, os trabalhos apresentados mostram resultados promissores a respeito de técnicas para conservação dos microverdes, no entanto, ressaltamos a necessidade da continuidade de pesquisas neste eixo temático, a fim de contribuir com o desenvolvimento científico, tecnológico e com a produção de alimentos saudáveis para a população.

\section{Agradecimentos}

Os autores agradecem à Fundação de Amparo à Pesquisa do Estado de Mato Grosso (FAPEMAT).

\section{Referências}

Alves, C. B. (2019). Como cultivar as Microgreens? Tudo o que você precisa saber! Moetá. https://www.moeta.eco.br/post/como-cultivar-as-microgreens-tudoo-que-voc\%C3\%AA-precisa-saber.

Baenas, N., Gómez-Jodar, I., Moreno, D. A., García-Viguera, C., \& Periago, P. M. (2017). Broccoli and radish sprouts are safe and rich in bioactive phytochemicals. Postharvest Biology and Technology, 127, 60-67.

Binder, G. (2012). Mighty Microgreens. College of Agriculture and Natural Resources. https://agnr.umd.edu/news/mighty-microgreens.

Canal do Horticultor. (2018). Como montar um sistema de baixo custo para a produção de Microverdes. https://canaldohorticultor.com.br/como-montar-umsistema-de-baixo-custo-para-a-producao-de-microverdes/

FAC - Perguntas frequêntes. (2016). MightyGreens. https://www.mightygreens.farm/faq.

Canal do Horticultor. (2021). Microverdes: Tendências da nova horticultura. https://canaldohorticultor.com.br/microverdes-tendencia-da-nova-horticultura/.

Chandra, D., Kim, J. G., \& Kim, Y. P. (2012). Changes in microbial population and quality of microgreens treated with different sanitizers and packaging films. Horticulture Environment and Biotechnology, 53, 32-40.

Choi, S.Y.; Chu, H. J.; Rajalingam, N.; Chae, H. B.; Yoon, J. H.; Hwang, I.; Kim, S. R. (2020). Growth of Escherichia coli and Listeria monocytogenes on radish microgreens washed with sodium hypochlorite during storage. Korean Journal of Food Preservation. https://www.ekosfop.or.kr/archive/view_article?pid=kjfp27-7-850.

Dalal, N., Siddiqui, S., \& Phogat, N. (2020). Post-harvest quality of sunflower microgreens as influenced by organic acids and ethanol treatment. Journal of Food Processing and Preservation, 14678, 01-09.

Di Bella, M. C., Toscano, S., Arena, D., Moreno, D. A., Romano, D., \& Branca, F. (2021). Effects of Growing Cycle and Genotype on the Morphometric Properties and Glucosinolates Amount and Profile of Sprouts, Microgreens and Baby Leaves of Broccoli (Brassica oleracea L. italica Plenck) and Kale (B. oleracea L. var. acephala DC.). Agronomy, 11, 1685.

Işık, H., Topalcengiz, Z., Güner, S., \& Aksoy, A. (2020). Generic and Shiga toxin-producing Escherichia coli (O157:H7) contamination of lettuce and radish microgreens grown in peat moss and perlite. Food Control, 111, 107079.

Katsenios, N., Christopoulus, M. V., Kakabouki, I., Vlachakis, D., Kavvadias, V., \& Efthimiadou, A. (2021). Effect of Pulsed Electromagnetic Field on Growth, Physiology and Postharvest Quality of Kale (Brassica oleracea), Wheat (Triticum durum) and Spinach (Spinacia oleracea) Microgreens. Agronomy, $11,1364$.

Kou, L.; Luo, Y.; Yang, T.; Xiao, Z.; Turner, E. R.; Lester, G. E.; Wang, Q.; Camp, M. J. (2013). Postharvest biology, quality and shelf life of buckwheat microgreens. LWT - Food Science and Technology. 51, 73-78.

Kyriacou, M. C., Rouphael, Y., Di Gioia, F., Kyratzis, A., Serio, F., Renna, M., De Pascale, S., \& Santamaria, P. (2016). Micro-scale vegetable production and the rise of microgreens. Trends in Food Science \& Technology, 57, 103-115.

Laurindo, J. (2020). Microverdes: conheça esse superalimento que pode ser utilizado em receitas do dia a dia. NSC Total. https://www.nsctotal.com.br/noticias/microverdes.

Lopez-Cervantes et al. (2013). Effect of biotic elicitors on isothiocyanate and other phytochemical levels in broccoli microgreens. International J of Food Sci and Technol. 48, 2267-2275.

Lu, Y., Dong, W., Alcanzar, J., Yang, T., Luo, Y., Wang, Q., \& Chen, P. (2018). Effffect of preharvest CaCl2 spray and postharvest UV-B radiation on storage quality of broccoli microgreens, a richer source of glucosinolates. Journal of Food Composition and Analysis, 67, 55-62.

Lu, Y., Dong, W., Yang, T., Luo, Y., \& Chen, P. (2021). Preharvest UVB application increases glucosinolate contents and enhances postharvest quality of broccoli microgreens. Molecules, 26, 3247.

Martins, C. (2019). Armazenamento de alimentos: por que fazer de modo correto. Instituto Cristina Martins. https://institutocristinamartins.com.br/armazenamento-de-alimentos-por-que-fazer-de-modo-correto-veja/. 
Research, Society and Development, v. 11, n. 3, e25211326584, 2022 (CC BY 4.0) | ISSN 2525-3409 | DOI: http://dx.doi.org/10.33448/rsd-v10i3.26584

Mir, S. A., Shah, M. A., \& Mir, M. M. (2016). Microgreens: Production, shelf life and bioactive components. Critical Reviews in Food Science and Nutrition. Muchjajib, U., Muchjajib, S., Suknikom, S., \& Butsai, J. (2015). Production of microgreens from local plant species in Thailand. Acta Horticulturea, 1091.

Paradiso, V. M., Castellino, M., Renna, M., Gattullo, C. E., Calasso, M., Terzano, R., Allegretta, I., Leoni, B., Caponio, F., \& Santamaria, P. (2018). Nutritional characterization and shelf-life of packaged microgreens. Food Function, 9, 5629-5640.

Photchanachai, S., Tantharapornrerk, N., Pola, W., Muangkote, S., \& Bayogan, E. R. V. (2018). Coconut coir media sterilization method for growing Chinese kale microgreens. Acta Horticulturea, 1210.

Reed, E., Ferreira, C. M., Bell, R., Brown, E. W., \& Zheng, J. (2018). Plant-Microbe and Abiotic Factors Influencing Salmonella Survival and Growth on Alfalfa Sprouts and Swiss Chard Microgreens. Applied and Environmental Microbiology, 84.

Renna, M., \& Paradiso, V. M. (2020). Ongoing Research on Microgreens: Nutritional Properties, Shelf-Life, Sustainable Production, Innovative Growing and Processing Approaches. Foods, 9, 826.

Revista Campo \& Negócios Hortifrúti. (2017). Microverdes - Projeto inédito no Brasil. https://revistacampoenegocios.com.br/microverdes-projeto-inedito-nobrasil/.

Rocchetti, G., Tomas, M., Zhang, L., Zengin, G., Lucini, L., \& Capanoglu, E. (2020). Red beet (Beta vulgaris) and amaranth (Amaranthus sp.) microgreens: Effect of storage and in vitro gastrointestinal digestion on the untargeted metabolomic profile. Food Chemistry, $332,127415$.

Santos, F. L. Dos. (2019). Diferentes substratos no desenvolvimento de microverdes de beterraba. Universidade Federal Da Fronteira Sul - UFFS. https://rd.uffs.edu.br/bitstream/prefix/3762/1/SANTOS.pdf.

Supapnavich, S., Sangsuk, P., Sripumimas, S., \& Anuchai, J. (2020). Efficiency of low dose cyanocobalamin immersion on bioactive compounds contents of ready to eat sprouts (sunflower and daiko) and microgreens (red-amaranth) during storage. Postharvest Biology and Technology, $160,111033$.

Turner, E. R., Luo, Y., \& Buchanan, R. L. (2020). Microgreen nutrition, food safety, and shelf life: A review. Journal of Food Science, 85, 870-882.

Vamos comer melhor. (2019). 6 passos para produzir microverdes. https://vamoscomermelhor.com.br/6-passos-para-produzir-microverdes/

Verlinden, S. (2020). Microgreens: Definitions, Product Types, and Production Practices. Horticultural Reviews, 47, 85-124.

Você sabe o que é Shelf Life? Saiba como pode ser calculado. (2021). Blog da Segurança Alimentar https://blogdasegurancaalimentar.volkdobrasil.com.br/shelf-life/\#.

Xiao, Z, Lester, G. E., Luo, Y., Xie, Z., Yu, L., \& Wang, Q. (2014b). Effect of light exposure on sensorial quality, concentrations of bioactive compounds and antioxidant capacity of radish microgreens during low temperature storage. Food Chemistry, 151, 472-479.

Xiao, Z., Luo, Y., Lester, G. E., Kou, L., Yang, T., \& Wang, Q. (2014a). Postharvest quality and shelf life of radish microgreens as impacted by storage temperature, packaging film, and chlorine wash treatment. LWT - Food Science and Technology. 55, 551-558. 